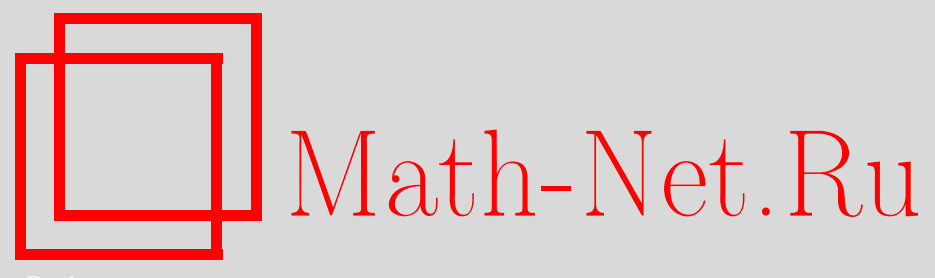

А. В. Тарасов, О свойствах функций, представимых в виде 2-КНФ, Дискрет. матем., 2001, том 13, выпуск 4, 99115

DOI: https://doi.org/10.4213/dm309

Использование Общероссийского математического портала Math-Net.Ru подразумевает, что вы прочитали и согласны с пользовательским соглашением http://www . mathnet.ru/rus/agreement

Параметры загрузки:

IP: 54.80 .73 .141

26 апреля 2023 г., 17:52:18 


\title{
О свойствах функций, представимых в виде 2-КНФ
}

\author{
(C) 2001 г. $\quad$ A. B. Тарасов
}

\begin{abstract}
В статье рассматриваются свойства булевых биюнктивных функций. Класс булевых биюнктивных функций состоит из функций, представимых в виде 2-КНФ. В работе построен алгоритм минимизации биюнктивных функций, исследуются некоторые свойства этих функций.
\end{abstract}

\section{1. Введение}

Одной из важных проблем дискретной математики является задача решения систем булевых уравнений. В общем случае, как известно $[1,3,4]$, данная задача является $N P$-трудной, а задача распознавания совместности таких систем - $N P$-полной. Однако, существует ряд классов систем булевых уравнений, задача распознавания совместности которых полиномиальна, наиболее известный из них - класс систем линейных булевых уравнений.

Введем понятие системы уравнений без ограничений на выбор неизвестных [4]. Пусть $F$ - произвольный конечный набор булевых функций, записанных булевыми формулами, $X=\left\{x_{1}, x_{2}, \ldots\right\}$ - счетное множество переменных. Через $[F]_{N C}$ обозначим класс систем уравнений вида

$$
f_{s_{i}}\left(x_{s_{i_{1}}}, \ldots, x_{s_{i_{n}}}\right)=1, \quad x_{s_{i_{j}}} \in X, \quad 1 \leqslant j \leqslant n, \quad f_{s_{i}} \in F, \quad i=1, \ldots, t .
$$

Такие системы называются системами без ограничений на выбор неизвестных [3, 4]. Задачу распознавания совместности таких систем обозначим через sat $\left([F]_{N C}\right)$. Понятие системы без ограничений на выбор неизвестных является аналогом понятия $s$-формулы, введенного Т. Шефером в [1], а задача sat $\left([F]_{N C}\right)$ - аналогом задачи выполнимости $s$-формулы. В [1] доказана теорема разделимости для задачи выполнимости $s$-формулы. В работах $[2,3]$ приведены ее эквивалентные формулировки. В нашей терминологии теорема выглядит следующим образом (см. [3]): если конечный набор функций $F$ такой, что любая функция $f \in F$ не равна тождественно нулю, целиком содержится в одном из классов булевых функций $0-S, 1-S, A, B i, W P, W N$, то задача $\operatorname{sat}\left([F]_{N C}\right)$ полиномиальна, в противном случае эта задача $N P$-полна. При этом 0-S - класс 0-выполнимых функций, 1-S - класс 1-выполнимых функций, $A-$ класс мультиаффинных функций, $B i$ - класс биюнктивных функций, $W P-$ класс слабс положительных функций, $W N$ - класс слабо отрицательных функций. Определения данных классов приведены в [1-3]. Если набор функций $F$ целиком 
содержится в классе биюнктивных функций, то задача $\operatorname{sat}\left([F]_{N C}\right)$ решается с линейной сложностью [2].

Проблематика систем без ограничения на выбор неизвестных исследовалась С. П. Горшковым в [3-5]. В частности, в [3] рассматриваются задачи распознавания совместности систем с произвольной правой частью и систем при задании некоторых ограничений на решения, введено понятие полиномиально решаемого класса систем уравнений, где под полиномиально решаемым классом понимается класс систем уравнений $R$, для которого задача $\operatorname{sat}(R)$ полиномиальна и существует эффективный алгоритм нахождения решений систем. Показано, что классы систем уравнений $[A]_{N C},[B i]_{N C},[W P]_{N C},[W N]_{N C}$ являются полиномиально решаемыми. В [4] доказана теорема разделимости для сложности задачи нахождения числа решений систем уравнений без ограничений на выбор неизвестных. В [5] рассматриваются пересечения классов $A, B i, W P, W N$. В [3] получены оценки для числа $b(n)$ биюнктивных функций от $n$ переменных

$$
n^{2} / 2 \leqslant \log _{2} b(n) \leqslant n^{2} .
$$

С. А. Гизуновым и В. А. Носовым в [6] описаны биюнктивные функции от трех и четырех переменных. Однако, более подробного изучения свойств самих биюнктивных функций до сих пор не проводилось. Данная статья ставит целью более глубокое изучение некоторых свойств биюнктивных функций.

Первый параграф настоящей статьи посвящен описанию алгоритма минимизации биюнктивных функций, записанных в виде 2-КНФ. Заметим, что задача минимизации произвольной булевой функции, заданной в виде дизъюнктивной нормальной форме (ДНФ) или конъюктивной нормальной форме (КНФ) является известной и сложной проблемой (см., например, [11]), однако для биюнктивных функций существует полиномиальный алгоритм минимизации. Второй параграф посвящен оценке сложности задачи нахождения числа выполняющих наборов биюнктивных функций, записанных в виде КНФ, а также доказательству неравновероятности биюнктивных функций при пяти и более существенных переменных.

\section{2. Представление биюнктивных функций графами}

\section{и их минимизация}

Пусть ненулевая булева биюнктивная функция $f\left(x_{1}, \ldots, x_{n}\right)$ задана в виде произвольной 2-КНФ:

$$
f\left(x_{1}, \ldots, x_{n}\right)=\bigotimes_{i=1}^{t}\left(x_{s_{i 1}}^{a_{i 1}} \vee x_{s_{i 2}}^{a_{i 2}}\right)
$$

где $x^{0}=\bar{x}$ и $x^{1}=x$. Аналогично тому, как это сделано в [2], определим ориентированный граф $G=(V, E)$, связанный с этой 2-КНФ, полагая

$$
\begin{aligned}
& V=\left\{x_{1}, \ldots, x_{n}, \overline{x_{1}}, \ldots, \overline{x_{n}}\right\}, \\
& E=\left\{\left(x_{s_{i 1}}^{a_{i 1} \oplus 1}, x_{s_{i 2}}^{a_{i 2}}\right),\left(x_{s_{i 2}}^{a_{i 2} \oplus 1}, x_{s_{i 1}}^{a_{i 1}}\right), i=1, \ldots, t\right\} .
\end{aligned}
$$

Для произвольной функции $f\left(x_{1}, \ldots, x_{n}\right)$ через $f_{i_{1} \ldots i_{k}}^{a_{1} \ldots a_{k}}$ будем обозначать ее подфункцию от $n-k$ переменных, полученную путем фиксации переменных $x_{i_{1}}, \ldots, x_{i_{k}}$ значениями $a_{1}, \ldots, a_{k}, 0 \leqslant k \leqslant n$. В дальнейшем будем рассматривать графы без петель и кратных дуг (см. [7, 9]). Сформулируем следующий результат. 
Теорема 1. Пусть ненулевая булева биюнктивная функция $f\left(x_{1}, \ldots, x_{n}\right)$ задана в виде 2-КНФ (1), а $G$ - граф этой 2-KНФ, построенный в соответствии с (2). Tогдa

- если в графе $G$ существуют ориентированные пути из $x_{i}^{a \oplus 1}$ в $x_{j}^{b}$ и из $x_{j}^{b \oplus 1}$ в $x_{i}^{a}$, то элементарная дизғюнкция $x_{i}^{a} \vee x_{j}^{b}$ является имплищентой функции $f$,

- если элементарная дизбюнкция $x_{i}^{a} \vee x_{j}^{b}$ является простой имплицентой функции $f$, заданной 2-КНФ (1), то в графе $G$ существуют ориентированные пуmи из $x_{i}^{a \oplus 1}$ в $x_{j}^{b}$ и из $x_{j}^{b \oplus 1}$ в $x_{i}^{a}$.

Доказательство. Сначала заметим, что в силу свойств графа $G$ из существования одного из указанных путей следует существование другого. Предположим, что указанные пути существуют. Пусть

$$
u_{0}=x^{a \oplus 1}, u_{1}, \ldots, u_{k}=x_{j}^{b}
$$

- один из путей, ведущих из $x_{i}^{a \oplus 1}$ в $x_{j}^{b}$. Тогда 2-КНФ (1) включает в себя произведение

$$
\left(x_{i}^{a} \vee u_{1}\right)\left(\bar{u}_{1} \vee u_{2}\right) \ldots\left(\bar{u}_{k-1} \vee x_{j}^{b}\right)
$$

Но тогда

$$
f \leqslant\left(x_{i}^{a} \vee u_{1}\right)\left(\bar{u}_{1} \vee u_{2}\right) \ldots\left(\bar{u}_{k-1} \vee x_{j}^{b}\right) \leqslant x_{i}^{a} \vee x_{j}^{b},
$$

где отношение $f \leqslant g$ для булевых функций означает, что $f \& g \equiv f$, то есть функция $g$ является имплицентой функции $f$. Следовательно, функция $x_{i}^{a} \vee x_{j}^{b}$ является имплицентой функции $f$.

Докажем обратное. Пусть $x_{i}^{a} \vee x_{j}^{b}$ - простая имплицента $f$. Положим $u=x_{i}^{a \oplus 1}$, $v=x_{j}^{b}$. Пусть $M_{1}$ и $M_{2}$ - множества вершин графа, заданные следующим образом:

$$
\begin{aligned}
& M_{1}=\{w \in V \mid \text { в } G \text { существует ориентированный путь из } u \text { в } w\} \\
& M_{2}=\{y \in V \mid \text { в } G \text { существует ориентированный путь из } y \text { в } v\} .
\end{aligned}
$$

Покажем сначала, что $M_{1} \neq \varnothing, M_{2} \neq \varnothing$. Пусть множество $M_{1}$ пусто. Это означает, что в формуле (1) отсутствует литерал $x_{i}^{a}$ и формулу (1) можно переписать в виде

$$
f\left(x_{1}, \ldots, x_{n}\right)=\ell_{i=1}^{r}\left(x_{i}^{a \oplus 1} \vee x_{q_{1}}^{c_{1}}\right) g\left(x_{1}, \ldots, x_{n}\right),
$$

где функция $g$ не зависит существенно от $x_{i}$. Но тогда $f_{i}^{a \oplus 1} \equiv g$. Поскольку $x_{i}^{a} \vee x_{j}^{b}$ - имплицента $f$, должно выполняться равенство

$$
f_{i j}^{a \oplus 1 b \oplus 1}=f\left(x_{1}, \ldots, x_{i-1}, a \oplus 1, x_{i+1}, \ldots, x_{j-1}, b \oplus 1, x_{j+1}, \ldots, x_{n}\right) \equiv 0 .
$$

Это означает, что

$$
g_{j}^{b \oplus 1}=g\left(x_{1}, \ldots, x_{j-1}, b \oplus 1, x_{j+1}, \ldots, x_{n}\right) \equiv 0
$$

а значит, функцию $g$ можно записать в виде

$$
g\left(x_{1}, \ldots, x_{n}\right)=x_{j}^{b} h\left(x_{1}, \ldots, x_{n}\right),
$$


и функция $h$ не зависит существенно от $x_{j}$. Но тогда

$$
f\left(x_{1}, \ldots, x_{n}\right)=\ell_{i=1}^{r}\left(x_{i}^{a \oplus 1} \vee x_{q_{i}}^{c_{i}}\right) x_{j}^{b} h,
$$

и, следовательно, $x_{j}^{b}$ является имплицентой $f$, что противоречит условию простоты имплиценты. Аналогично показывается, что $M_{2} \neq \varnothing$.

Для доказательства теоремы достаточно показать, что $M_{1} \cap M_{2} \neq \varnothing$. Для $w \in M_{1}$ пусть $g_{w}$ обозначает произведение дизъюнктов из правой части (1), соответствующих дугам всех путей из $u$ в $w$, а для $y \in M_{2}$ пусть $h_{y}-$ произведение дизъюнктов, соответствующих дугам всех путей из $y$ в $v$. Легко видеть, что для $w \in M_{1}$ и $y \in M_{2}$ имеют место соотношения

$$
g_{w} \geqslant f, \quad h_{y} \geqslant f, \quad\left(g_{w}\right)_{i}^{a \oplus 1} \equiv w, \quad\left(h_{y}\right)_{j}^{b \oplus 1} \equiv y .
$$

Тогда

$$
f\left(x_{1}, \ldots, x_{n}\right)=\underset{w \in M_{1}}{\ell} g_{w} \underset{y \in M_{2}}{\bigotimes} h_{y} \& p\left(x_{1}, \ldots, x_{n}\right),
$$

где $p\left(x_{1}, \ldots, x_{n}\right) \geqslant f$ и $p\left(x_{1}, \ldots, x_{n}\right)$ - произведение сомножителей из $(1)$, не входящих в $g_{w}$ и $h_{y}, w \in M_{1}, y \in M_{1}$. Очевидно, что

$$
p_{i j}^{a \oplus 1 b \oplus 1}=p\left(x_{1}, \ldots, x_{i-1}, a \oplus 1, x_{i+1}, \ldots, x_{j-1}, b \oplus 1, x_{j+1}, \ldots, x_{n}\right) \neq 0 .
$$

Тогда

$$
f_{i j}^{a \oplus 1 b \oplus 1}=\underset{w \in M_{1}}{\bigotimes} w \underset{y \in M_{2}}{\bigotimes} \bar{y} \& p_{i j}^{a \oplus 1 b \oplus 1} \equiv 0
$$

поскольку $x_{i}^{a} \vee x_{j}^{b}$ - имплицента $f$. Для доказательства теоремы достаточно показать, что

$$
\underset{w \in M_{1}}{\bigotimes} w \neq \equiv 0, \quad \underset{t \in M_{2}}{\&} \bar{y} \not \equiv, \quad \underset{w \in M_{1}}{\bigotimes} \underset{y \in M_{2}}{\&} \bar{y} \equiv 0
$$

Пусть

$$
p_{i j}^{a \oplus 1 b \oplus 1}=\bigotimes_{q=1}^{r}\left(x_{m_{q 1}}^{d_{q 1}} \vee x_{m_{q 2}}^{d_{q 2}}\right)
$$

Рассмотрим следующие три случая.

Пусть функция $p_{i j}^{a \oplus 1 b \oplus 1}$ не зависит существенно от переменных, входящих в множества $M_{1}$ и $M_{2}$. В этом случае очевидно, что

$$
\underset{w \in M_{1}}{\&} w \underset{y \in M_{2}}{\ell} \bar{y} \equiv 0
$$

и теорема доказана.

Пусть $x_{m_{q 1}}^{d_{q 1} \oplus 1} \in M_{1}$ для некоторого $q, 1 \leqslant q \leqslant r$. Тогда $x_{m_{q^{2}}}^{d_{q_{2}}} \in M_{1}$ по определению множества $M_{1}$, что противоречит построению функции $p$.

Пусть $x_{m_{q 1}}^{d_{q 1}} \in M_{1}, 1 \leqslant q \leqslant r$. Из рассмотрений второго случая следует, что литерал $x_{m_{q 1}}^{d_{11} \oplus 1}$ не входит в построенную КНФ функции $p_{i j}^{a \oplus 1 b \oplus 1}$. Поэтому в формуле 
(3) можно опустить соответствующие сомножители и считать, что мы находимся в условиях первого случая.

Аналогичные рассуждения верны относительно множества $M_{2}$.

Поэтому формула (3) принимает вид

$$
\underset{w \in M_{1}}{\ell} w \underset{y \in M_{2}}{\ell} \bar{y} \& h \equiv 0 \text {, }
$$

причем функция $h$ не зависит существенно от переменных, соответствующих вершинам графа из множеств $M_{1}$ и $M_{2}$, а значит,

$$
\underset{w \in M_{1}}{\bigotimes} w \underset{y \in M_{2}}{\bigotimes} \bar{y} \equiv 0 \text {. }
$$

Ввиду того, что $\&_{w \in M_{1}} w \not \equiv 0$ и $\&_{y \in M_{2}} \bar{y} \not \equiv 0$, получаем, что $M_{1} \cap M_{2} \neq \varnothing$, что и требовалось доказать.

Имплицента булевой функции называется простой, если никакая ее собственная часть не является имплицентой исходной функции. Сокращенной КНФ булевой функции называется конъюнкция всех ее простых имплицент.

Введем несколько понятий из теории графов (см. $[7,8])$. Пусть $G=(V, E)-$ произвольный орграф без петель и кратных дуг. Как и в [8, гл. 5], транзитивным замыканием графа $G$ назовем граф $G^{T}=\left(V, E^{T}\right)$ такой, что для любых $u, v \in V$ ребро $(u, v) \in E^{T}$ тогда и только тогда, когда в графе $G$ существует ориентированный путь из вершины $u$ в вершину $v$. Очевидно, что в этом случае граф $G^{T}$ является транзитивным и его матрица смежности и матрица достижимости совпадают. Таким образом, справедливо следующее следствие доказанной теоремы.

Следствие 1. Если в условиях теоремы 1 функция $f$ задана сокращенной КНФ, то ее граф является транзитивным. Кроме того, он является транзитивным замыканием графа любой другой 2-KНФ, реализующей данную функцию.

Определение 1. Графы $G_{1}$ и $G_{2}$ будем называть транзитивно эквивалентными, если $G_{1}^{T}=G_{2}^{T}$. В этом случае будем писать $G_{1} \sim G_{2}$.

Очевидно, что введенное отношение является отношением эквивалентности и разбивает все множество графов с заданным множеством вершин на классы эквивалентности. Кроме того, в силу доказанных утверждений, если графы двух различных 2-КНФ транзитивно эквивалентны, то обе 2-КНФ задают одну и ту же биюнктивную функцию.

Класс эквивалентности графа $G$ будем обозначать через $[G]$. Очевидна следующая лемма.

Лемма 1. Пусть $G=(V, E)-$ орграф, не содержащий дуги $(u, v)$, но содержащий ориентированный путь из и в $v$. Положим $G^{\prime}=\left(V, E^{\prime}\right)$, где $E^{\prime}=E \cup\{(u, v)\}$. Тогда $G^{\prime} \in[G]$.

Если заданы два графа $G_{1}=\left(V, E_{1}\right)$ и $G_{2}=\left(V, E_{2}\right)$, то их пересечением называется граф $G_{1} \cap G_{2}=\left(V, E_{1} \cap E_{2}\right)$ (см. [7, гл. 2]). Для заданного орграфа $G$ определим граф $G_{R}=L_{H \in[G]} H$. Дуги этого графа назовем ядровыми дугами графа $G$. Орграф назовем ациклическим, если он не имеет орциклов, и связным, если он связен как неориентированный граф [7]. 
Лемма 2. Если $G$ - ацихлический орграф, то $G_{R} \in[G]$. Если $G$ - связный орграф c $n \leqslant 3$ вершинами и $G_{R} \in[G]$, то $G$ ациклический.

Доказательство. Пусть $G$ - ациклический граф, содержащий хотя бы один ориентированный путь из вершины $u$ в вершину $v$. Выберем наиболее длинный из этих путей (или один из наиболее длинных, если таких несколько). Обозначим его вершины $u=w_{0}, \ldots, w_{t}=v$. Для произвольного $H \in[G]$ покажем, что дуги $\left(w_{0}, w_{1}\right), \ldots,\left(w_{t-1}, w-t\right)$ лежат в множестве дуг графа $H$. Если $t=1$, то дуга $(u, v)$ - единственный путь в $G$ из $u$ в $v$. Значит, по определению графа $G^{T}$, она будет единственным путем из $u$ в $v$ в графе $G^{T}$, а следовательно, и в $H$. Пусть $t>1$. Покажем, что дуга $\left(w_{i-1}, w_{i}\right)$ является самым длинным путем из $w_{i-1}$ в $w_{i}$, $1 \leqslant i \leqslant t$. Предположим противное, а именно, пусть существует ориентированный путь $w_{i-1}, u_{1}, \ldots, u_{k}, w_{i}$.

В этом случае, если множество вершин $\left\{u_{1}, \ldots, u_{k}\right\}$ не содержит вершин из $\left\{w_{0}, \ldots, w_{t}\right\}$, то приходим к противоречию с тем, что путь $w_{0}, \ldots, w_{t}-$ самый длинный путь. Значит, указанные множества пересекаются. Пусть $w_{j} \in\left\{u_{1}, \ldots, u_{k}\right\}$. Тогда возможны два случая: либо $j<i-1$, и тогда получаем, что вершины $w_{i-1}$ и $w_{j}$ взаимно достижимы, либо $j>i$ и взаимно достижимыми будут вершины $w_{i}$ и $w_{j}$. В обоих случаях получаем противоречие с условием ацикличности графа. Поэтому рассуждения, аналогичные случаю $t=1$ можно провести для дуг $\left(w_{0}, w_{1}\right), \ldots,\left(w_{t-1}, w_{t}\right)$. Первая часть леммы доказана.

Предположим теперь, что $G_{R} \in[G]$ и граф $G$ имеет орцикл. Выберем в нем максимальный по включению цикл, являющийся, очевидно, сильно связной компонентой графа. Пусть $w_{1}, \ldots, w_{t-1}, w_{t}-$ множество вершин этого цикла и $t>2$. Построим графы $H_{1}$ и $H_{2}$ следующим образом: для построения $H_{1}$ в графе $G$ заменим все дуги цикла на дуги $\left(w_{1}, w_{2}\right), \ldots,\left(w_{t-1}, w_{t}\right),\left(w_{t}, w_{1}\right)$, а для построения графа $H_{2}$ на дуги $\left(w_{1}, w_{t}\right),\left(w_{t}, w_{t-1}\right) \ldots,\left(w_{2}, w_{1}\right)$. Очевидно, что $H_{1}, H_{2} \in[G]$. Из определения графа $G_{R}$ получаем, что в нем не может быть цикла на вершинах $w_{1}, \ldots, w_{t-1}, w_{t}$. В частности, вершины $w_{1}$ и $w_{2}$ не являются взаимно достижимыми, откуда следует, что $G_{R} \notin[G]$, что и требовалось доказать. Пусть далее $t=2$. В силу связности графа $G$ найдется вершина $w_{3}$, смежная с одной из вершин $w_{1}$ и $w_{2}$, такая, что соответствующая дуга является наидлиннейшим путем. Пусть, например, $\left(w_{1}, w_{3}\right)-$ такая дуга. Тогда в $G^{T}$ есть дуги $\left(w_{1}, w_{2}\right)$ и $\left(w_{2}, w_{3}\right)$. Построим граф

$$
H=\left(V, E_{1}\right), \quad E_{1}=E \backslash\left\{\left(w_{1}, w_{3}\right)\right\} \cup\left\{\left(w_{2}, w_{3}\right)\right\}
$$

Легко видеть, что в графе $G \cap H$ вершина $w_{3}$ недостижима из вершин $w_{1}$ и $w_{2}$, а значит, $G_{R} \notin[G]$. Лемма доказана.

Если мы рассмотрим произвольный связный орграф на двух вершинах, каких всего два, то увидим, что всегда $G_{R} \in[G]$.

Определение 2. Пусть граф $G$ такой, что $G_{R} \in[G]$, тогда граф $G_{R}$ назовем его минимизацией.

Определение 3. Пусть $G$ - орграф, $Y_{1}, \ldots, Y_{m}$ - его сильно связные компоненты. Конденсацией графа $G$ назовем граф $R(G)=(V R(G), E R(G))$ такой, что

$$
V R(G)=\left\{Y_{1}, \ldots, Y_{m}\right\}
$$


и

$$
\left(Y_{i}, Y_{j}\right) \in E R(G) \Longleftrightarrow u \in Y_{i}, v \in Y_{j}:(u, v) \in E
$$

(см., например, [9, гл. 2]).

Легко показать, что $R(G)$ - ациклический орграф.

Для произвольного графа $G$ через $M(G)$ обозначим множество графов из $[G]$, имеющих минимальное число дуг. Если $G$ - ациклический граф, то очевидно, что $M(G)=\left\{G_{R}\right\}$.

Теорема 2. Множество графов $M(G)$ может быть построено следующим образом.

(1) Найти силъно связные компонентъ $Y_{1}, \ldots, Y_{m}$ графа $G$.

(2) Построить граф $R(G)_{R}$.

(3) Пусть $Y_{i}=\left\{x_{i_{1}}, \ldots, x_{i_{k_{i}}}\right\}, i=1,2, \ldots$, m. Построить произвольный цикл на множестве вершин $\left\{x_{i_{1}}, \ldots, x_{i_{k_{i}}}\right\}$.

(4) Для каждого варианта совокупности циклов и каждой дуги

$$
\left(Y_{i}, Y_{j}\right) \in E R(G), \quad Y_{i}=\left\{x_{i_{1}}, \ldots, x_{i_{k_{i}}}\right\}, \quad Y_{j}=\left\{x_{j_{1}}, \ldots, x_{j_{k_{j}}}\right\}, \quad i \neq j
$$

произволънъм образом выбратъ дугу $\left(x_{i_{p}}, x_{j_{q}}\right), 1 \leqslant p \leqslant k_{i}, 1 \leqslant q \leqslant k_{j}$.

Доказательство. Предположим, что $H$ - один из орграфов, построенных согласно пунктам 1-4. Если вершина $v$ достижима из вершины $u$ в графе $G$, то она достижима из $u$ и в графе $H$ согласно его построению. Обратное также очевидно, откуда следует, что $H \in[G]$.

Докажем, что построенные графы имеют наименьшее возможное число дуг среди графов из $[G]$. Пусть $H \in M(G)$. Дуги графа $H$ можно разделить на два множества: к первому отнесем дуги, соединяющие между собой вершины из одной сильно связной компоненты, а ко второму дуги, соединяющие вершины из различных компонент. Очевидно, что сильно связный граф с минимальным числом дуг - это цикл, поэтому первое множество дуг соответствует дугам одной из построенных в пункте 3 совокупностей циклов. Далее, поскольку граф имеет минимальное число дуг, любые сильно связные компоненты не могут быть соединены более, чем одной дугой, что соответствует пункту 4. Теорема доказана.

Перейдем к проблеме минимизации 2-КНФ.

Теорема 3. Пусть функция $f$ задана 2-КНФ (1), а $G$ - ее граф, заданный в (2). Тогда множество графов $H=\left(V, E_{H}\right)$ из $[G]$, обладающих свойством

$$
(u, v) \in E_{H} \Longleftrightarrow(\bar{v}, \bar{u}) \in E_{H}
$$

задает все 2-КНФ, реализующие функцию $f$.

Графы из $M(G)$, обладающие свойством (4), задают все минималънъе 2-КНФ функции $f$. В частности, если $G$ - ациклический граф, то граф $G_{R}$ является графом единственной минимальной 2-КНФ функции $f$. 
Доказательство. Докажем первое утверждение. В силу условий (2) для любого графа, реализующего 2-КНФ, выполняется свойство (4). Обратно, если указанное свойство выполнено, то по графу легко можно построить соответствующую 2-КНФ. В силу теоремы 1 всякий граф, реализующий некоторую $2-\mathrm{KH \Phi} \mathrm{функции} f$, лежит в $[G]$.

Перейдем к доказательству второго утверждения. Заметим, что графы из $M(G)$, обладающие свойством (6), задают некоторое множество 2-КНФ функции $f$. Поскольку эти графы имеют минимальное количество дуг среди графов из $[G]$, они являются графами минимальных 2-КНФ. Вторая часть этого утверждения непосредственно следует из первой. Теорема доказана.

В результате можно построить алгоритм минимизации биюнктивных функций следующего вида.

\section{Алгоритм 1.}

Вход: биюнктивная функция, заданная 2-КНФ (1).

Выход: минимальная 2-КНФ функции $f$.

Метод: 1 . Построить граф $G=(V, E)$ для 2-КНФ (1) согласно формуле (4).

2. В графе $G$ с помощью алгоритма 5.4 из [8, гл. 5] найти сильно связные компоненты.

3. Построить граф $H$ из $M(G)$, обладающий свойством (4), следующим образом.

3.1. Упорядочить вершины, входящие в сильно связные компоненты

$$
Y_{i}=\left\{x_{i_{1}}^{a_{i_{1}}}, \ldots, x_{i_{k_{i}}}^{a_{a_{k_{i}}}}\right\}, \quad i_{1}<i_{2}<\ldots<i_{k_{i}}, \quad 1 \leqslant i \leqslant m .
$$

3.2. Построить простые циклы на множествах $Y_{1}, \ldots, Y_{m}$ по следующему правилу: если $Y_{i}=\left\{x_{i_{1}}^{a_{i_{1}}}, \ldots, x_{i_{k_{i}}}^{a_{i_{i}}}\right\}$ и $a_{i_{1}}=1$, то образовать цикл $x_{i_{1}}^{a_{i_{1}}}, \ldots, x_{i_{k_{i}}}^{a_{i k_{i}}}$, в противном случае образовать цикл $x_{i_{k_{i}}}^{a_{i_{k_{i}}}}, \ldots, x_{i_{1}}^{a_{i_{1}}}$.

3.3. Если в графе $R(G)$ есть дуга $\left(Y_{i}, Y_{j}\right)$, то в граф $H$ добавить дугу $\left(x_{i_{1}}^{a_{i_{1}}}, x_{j_{1}}^{a_{j_{1}}}\right)$.

4. По графу $H$ построить минимальную 2-КНФ.

Теорема 4. Алгоритм 1 строит минимальную 2-КНФ биюнктивной бункции $f\left(x_{1}, \ldots, x_{n}\right)$ за $T(n)=O\left(n^{2}\right)$ операций.

Доказательство. Сначала надо показать, что алгоритм корректен, то есть что в результате его работы мы построим граф некоторой 2-КНФ функции $f$. В самом деле, согласно п.п. 3.2 и 3.3 в графе $H$ выполнено свойство (4). Следовательно, этот граф является графом некоторой 2-КНФ.

Трудоемкость 1-го этапа линейно зависит от длины КНФ. Но 2-КНФ может содержать не более $2 n(n-1)$ различных сомножителей. Следовательно, в графе будет не более $4 n(n-1)$ дуг. По теореме 5.5 из [7, гл. 5] трудоемкость 2-го этапа составит $O(\max \{n, t\})$ операций, где $t$ - число дуг графа. Далее, 3-й этап также можно сделать с линейной сложностью. Теорема доказана. 
Заметим, что если биюнктивная функция имеет аффинные или линейные имплиценты, существенно зависящие от двух переменных, то граф любой ее 2-КНФ имеет сильно связные компоненты. Если аффинная имплицента существенно зависит от одной переменной, то в графе есть ориентированный путь из некоторой вершины $u$ в $\bar{u}$. Кроме того, как показано в [5], если биюнктивная функция является 0- и 1-выполнимой, то она лежит в пересечении классов $W P$ и $W N$. Ряд свойств таких функций исследовался в [5].

В заключение данного параграфа рассмотрим вопрос о максимальной длине минимальной 2-КНФ.

Теорема 5. Пусть $f\left(x_{1}, \ldots, x_{n}\right)$ - биюнхтивная функция, не имеющая аффинных имплицент, а $t(f)$ - длина ее минимальной $2-K Н \Phi$. Тогда если $f(1, \ldots, 1)=1$ и $f(0, \ldots, 0)=1$, mо $t(f) \leqslant\left\lfloor n^{2} / 4\right\rfloor$, в противном случае $t(f) \leqslant\left(\begin{array}{c}n \\ 2\end{array}\right)$.

Обе указанные оченки достижимы.

Доказательство. По условию теоремы граф $G$ минимальной $2-$ КНФ функции $f$ не имеет нетривиальных сильно связных компонент. Докажем первую оценку. Если $f(1, \ldots, 1)=1$ и $f(0, \ldots, 0)=1$, то граф $G$ можно разбить на два непересекающихся подграфа: один на множествах вершин $x_{1}, \ldots, x_{n}$, а другой на множестве их отрицаний. Сужение графа $G$ на множестве вершин $x_{1}, \ldots, x_{n}$ обозначим через $H$. Предположим, что функция $f$ имеет самую длинную миннмальную 2-КНФ длины $t$ в классе функций, удовлетворяющих условиям $f(1, \ldots, 1)=1$ и $f(0, \ldots, 0)=1$. Тогда граф $H$ имеет $t$ дуг.

Пусть $x_{i}-$ проходная вершина графа $H$. Положим

$$
\begin{aligned}
R^{+}\left(x_{i}\right) & =\left\{x_{j}:\left(x_{j}, x_{i}\right) \in E H\right\}, & R^{-}\left(x_{i}\right) & =\left\{x_{j}:\left(x_{i}, x_{j}\right) \in E H\right\} \\
r^{+}\left(x_{i}\right) & =\left|R^{+}\left(x_{i}\right)\right|, & r^{-}\left(x_{i}\right) & =\left|R^{-}\left(x_{i}\right)\right| .
\end{aligned}
$$

Положим $H^{\prime}=H \backslash\left\{x_{i}\right\}$, и пусть $t^{\prime}-$ число дуг графа $H^{\prime}$. Очевидно, что $H^{\prime}$ - граф минимальной 2-КНФ для некоторой функции $f^{\prime}$ от $n-1$ переменной, удовлетворяющей условиям $f(1, \ldots, 1)=1$ и $f(0, \ldots, 0)=1$. Тогда

$$
t=t^{\prime}+r^{+}\left(x_{i}\right)+r^{-}\left(x_{i}\right) \text {. }
$$

Произведем следующее преобразование графа $H$ : удалим дуги, инцидентные вершине $x_{i}$, после чего в полученный граф добавим всевозможные дуги вида $(u, v)$, где $u \in R^{+}\left(x_{i}\right), v \in R^{-}\left(x_{i}\right)$, а также дуги вида $\left(x_{i}, v\right), v \in R^{-}\left(x_{i}\right)$. Легко видеть, что полученный граф будет своей же минимизацией и является графом минимальной 2-КНФ некоторой биюнктивной функции. Кроме того, данный граф будет иметь $t^{\prime}+r^{+}\left(x_{i}\right) r^{-}\left(x_{i}\right)+r^{-}\left(x_{i}\right)$ дуг. Так как $x_{i}-$ проходная вершина, то $r^{+}\left(x_{i}\right)>0$, $r^{-}\left(x_{i}\right)>0$, а значит, число дуг этого графа строго больше, чем $t$. Следовательно, если функция $f$ имеет минимальную 2-КНФ максимальной длины, то граф этой 2 -КНФ не имеет проходных вершин. Если же граф $H$ имеет $k$ начальных и $n-k$ конечных вершин, то максимальное число дуг в нем равно $k(n-k)$. Как известно, максимум этого выражения достигается при $k=\lfloor n / 2\rfloor$ и равен $\left\lfloor n^{2} / 4\right\rfloor$ в случае, когда, например,

$$
f\left(x_{1}, \ldots, x_{n}\right)=\bigotimes_{i=1}^{\lfloor n / 2\rfloor} \bigotimes_{j=\lfloor n / 2\rfloor+1}^{n}\left(\bar{x}_{i} \vee x_{j}\right)
$$


При доказательстве второй оценки аналогично предыдущему доказательству можно показать, что граф минимальной 2-КНФ не имеет проходных вершин. Однако, в этом случае максимальное возможное число дуг такого графа будет равно $\left(\begin{array}{l}n \\ 2\end{array}\right)$. В данном случае верхняя оценка достигается на функции вида

$$
f\left(x_{1}, \ldots, x_{n}\right)=\bigotimes_{i \neq j}\left(x_{i} \vee x_{j}\right)
$$

Теорема доказана.

Итак, мы показали, что задача минимизации биюнктивных функций, записанных произвольной 2-КНФ, полиномиальна и оценили сложность соответствующего алгоритма. В следующем параграфе мы исследуем свойства множеств выполняющих векторов биюнктивных функций.

\section{3. Свойства множеств выполняющих векторов биюнктивных функций}

Одной из важных характеристик булевой функции является ее вес, то есть число векторов, на которых функция принимает значение 1. Если булева биюнктивная функция задана в виде 2-КНФ вида

$$
f\left(x_{1}, \ldots, x_{n}\right)=\ell_{i=1}^{t}\left(x_{s_{i 1}}^{a_{i 1}} \vee x_{s_{i 2}}^{a_{i 2}}\right),
$$

то ее вес равен числу решений системы

$$
x_{s_{i 1}}^{a_{i 1}} \vee x_{s_{i 2}}^{a_{i 2}}=1, \quad i=1, \ldots, t .
$$

Сложность задачи нахождения числа решений систем булевых уравнений исследовалась в $[3,4]$. В соответствии с принятыми в этих работах обозначениями, задачу определения числа выполняющих векторов обозначим через enu $(R)$, где $R$ - класс систем вида (6). Данная задача, как и задачи определения числа решений произвольных булевых систем уравнений, относится к классу задач перечисления. Аксиоматика теории задач перечисления и соответствующая терминология, в частности, понятие \#Р-полноты, введены в п. 7.3 монографии [10].

В $[3,4]$ показано, что в общем случае задача определения числа решений систем уравнений, в частности, эквивалентная рассматриваемой задаче задача определения веса биюнктивной функции (в общем случае), является \#P-полной. Задача определения веса биюнктивной функции остается \#P-полной даже, если мы ограничиваемся случаями монотонных биюнктивных функций или функциями, являющимися одновременно слабо положительными и слабо отрицательными (класс таких функций в соответствии с [5] обозначим через $M_{3}$ ).

Введем новый класс задач перечисления. Через еnu $(r, k)$ обозначим задачу перечисления выполняющих наборов (в смысле подсчета их числа) произвольной системы уравнений вида

$$
x_{s_{i 1}}^{a_{i 1}} \vee x_{s_{i 2}}^{a_{i 2}} \vee \ldots \vee x_{s_{i r}}^{a_{i r}}=1, \quad i=1, \ldots, t,
$$

где каждое неизвестное встречается в системе не более $k$ раз. Нас интересует случай $r=2$. Верна следующая теорема. 
Теорема 6. Задача епu $(2, k)$ полиномиальна при $k \leqslant 2$ \# \#-полна в противном случае.

Доказательство. Пусть задана индивидуальная задача из еnu $(2, k)$. Будем считать, что исходная система имеет вид (6). Естественным образом построим функцию $f$, задав ее 2-КНФ (5). Произведя минимизацию этой 2-КНФ, мы все равно остаемся в условиях задачи еnu $(2, k)$, поскольку, исходя из свойств алгоритма минимизации из раздела 1 , каждая переменная будет встречаться в минимальной КНФ не более $k$ раз. Поэтому можно считать, что система (6) задает минимальную 2-КНФ. Докажем теорему для случая $f \in M_{3}$. Пусть $G(f)$ - граф минимальной 2-КНФ (5) функции $f\left(x_{1}, \ldots, x_{n}\right)$ и каждая переменная входит в 2-КНФ (5) не более двух раз. Рассмотрим граф $G(f)$ как неориентированный и для простоты будем считать, что он состоит из одной компоненты связности. В силу того, что каждая переменная входит в 2-КНФ не более двух раз, каждая вершина графа имеет степень не более 2. Это значит, что $G(f)$ является либо цепью, либо циклом.

Сначала рассмотрим случай, когда $G(f)$ - цепь. Вернемся к ориентации дуг графа $G(f)$. Легко видеть, что в этом случае граф $G(f)$ представляет собой сумму ориентированных путей. Пусть эти пути имеют длины

$$
l_{1}, \ldots, l_{k}, \quad l_{1}+\ldots+l_{k}=n-l .
$$

Через $N\left(l_{1}, \ldots, l_{k}\right)$ обозначим вес функции $f$, который будем обозначать $\|f\|$.

Для удобства будем считать, что неориентированный граф $G(f)$ является цепью вида $x_{1}, x_{2}, \ldots, x_{n-1}, x_{n}$ и что в ориентированном графе $G(f)$ вершина $x_{n}$ является начальной. Тогда

$$
\|f\|=\left\|f_{n}^{0}\right\|+\left\|f_{n}^{1}\right\| .
$$

Вершина $x_{n}$ является начальной вершиной ориентированного пути длины $l_{k}$. Если $l_{k}>l$, то

$$
\left\|f_{n}^{0}\right\|=N\left(l_{1}, \ldots, l_{k}-1\right), \quad\left\|f_{n}^{1}\right\|=N\left(l_{1}, \ldots, l_{k-1}-1\right)
$$

в силу основных свойств функции из класса $M_{3}$. Если же $l_{k}=l$, то

$$
\left\|f_{n}^{1}\right\|=N\left(l_{1}, \ldots, l_{k-1}-1\right), \quad\left\|f_{n}^{0}\right\|=N\left(l_{1}, \ldots, l_{k-1}\right) .
$$

Положив $N\left(l_{1}, \ldots, l_{k-1}, 0\right)=N\left(l_{1}, \ldots, l_{k-1}\right)$, получим равенство

$$
N\left(l_{1}, \ldots, l_{k}\right)=N\left(l_{1}, \ldots, l_{k}-1\right)+N\left(l_{1}, \ldots, l_{k-1}-1\right) .
$$

Заметим, что $N\left(l_{1}\right)=l_{1}+2$. Далее, пользуясь полученной формулой (7), можно за полиномиально зависящее от $n=l_{1}+\ldots+l_{k}+1$ число операций вычислить значение $N\left(l_{1}, \ldots, l_{k}\right)$. Алгоритм в данном случае состоит в последовательном вычислении значений

$$
N\left(l_{1}\right), N\left(l_{1}, 1\right), \ldots, N\left(l_{1}, l_{2}\right), \ldots, N\left(l_{1}, \ldots, l_{k}\right) .
$$

Каждая последующая величина вычисляется путем применения формулы (7) к некоторым из уже вычисленных ранее величин.

Теперь рассмотрим случай, когда неориентированный граф $G(f)$ является циклом. Пусть этот цикл имеет вид $x_{1}, x_{2}, \ldots, x_{n-1}, x_{n}, x_{1}$. В случае, когда этот цикл является сильно связной компонентой, задача тривиальна. Предположим противное. Тогда орграф $G(f)$ содержит либо дугу $\left(x_{1}, x_{n}\right)$, либо противоположную ей. 
Рассмотрим первый случай, так как второй рассматривается аналогично. В этом случае имеет место равенство

$$
f\left(x_{1}, \ldots, x_{n}\right)=g\left(x_{1}, \ldots, x_{n}\right) \&\left(\overline{x_{1}} \vee x_{n}\right)
$$

В силу очевидных соображений выполнено равенство $\|f\|=\|g\|-\|h\|$, где $h=g_{1, n}^{1,0}$. Но функции $g$ и $h$ принадлежат классу $M_{3}$, а неориентированные графы $G(g)$ и $G(h)$ являются цепями. Тогда, в соответствии с первой частью доказательства, веса этих функций вычислимы за полиномиальное время. Значит, за полиномиальное время вычисляется вес функции $f$.

В случае, когда граф $G(f)$ не связен, он распадается на компоненты связности, являющиеся либо цепями, либо циклами. Вычислив веса функций, задаваемых компонентами связности, нам достаточно их перемножить. Легко видеть, что трудоемкость расчета веса в нашем случае есть $O\left(n^{2}\right)$.

Рассмотрим теперь общий случай. Пусть в 2-КНФ (5) каждая переменная встречается не более двух раз. Это значит, что в графе $G$ каждая вершина имеет степень не более 2 . Тогда граф $G$ распадается на компоненты связности, являющиеся либо путями, либо циклами (неориентированными). Заметим следующий очевидный факт: если компонента связности $G^{\prime}=\left(V^{\prime}, E^{\prime}\right)$ содержит некоторую вершину вместе с инверсной к ней, то такая компонента является неориентированным циклом, причем $V^{\prime}=X^{\prime} \cup \bar{X}^{\prime}$, где $X^{\prime} \subset X=\left\{x_{1}, \ldots, x_{n}\right\}$. Для компонент, не содержащих пар инверсных вершин, мы можем воспользоваться первой частью доказательства теоремы, инвертировав некоторые переменные. Будем считать, что граф состоит из одной компоненты, являющейся неориентированным циклом. Длина минимальной $2-$ КНФ в этом случае составит $2 n+2$. Поскольку функция $f$ не равна тождественно 0, существует вектор $a=\left(a_{1}, \ldots, a_{n}\right)$ такой, что $f\left(a_{1}, \ldots, a_{n}\right)=1$. В силу результатов из [2-4], такой вектор строится за $O(n)$ операций. Функция $g=f\left(x_{1} \oplus a_{1} \oplus 1, \ldots, x_{n} \oplus a_{n} \oplus 1\right)$ имеет тот же вес, что и функция $f$, но является слабо положительной биюнктивной функцией, поскольку $g(1, \ldots, 1)=1$. Это значит, что функцию $g$ можно представить в виде

$$
g\left(x_{1}, \ldots, x_{n}\right)=\ell_{j=1}^{k}\left(x_{r_{j 1}} \vee \bar{x}_{r_{j 2}}\right) \bigotimes_{j=k+1}^{m}\left(x_{r_{j 1}} \vee x_{r_{j 2}}\right)
$$

Покажем, что в этой формуле $m-k=l$. В самом деле, поскольку граф минимальной 2-КНФ функции $g$ является неориентированным циклом, между множествами $X$ и $\bar{X}$ может проходить не более двух дуг. В противном случае найдутся вершины, имеющие степень более 2, что противоречит условию. В силу высказанных соображений получаем, что $g_{r_{m 1} r_{m 2}}^{00} \equiv 0$. Для удобства обозначений будем считать, что $r_{m 1}=1$, $r_{m 2}=n$. Тогда

$$
g\left(x_{1}, \ldots, x_{n}\right)=g^{\prime}\left(x_{1}, \ldots, x_{n}\right) \&\left(x_{1} \vee x_{n}\right),
$$

где функция $g^{\prime}\left(x_{1}, \ldots, x_{n}\right)$ принадлежит классу функций $M_{3}$. Отсюда следует, что

$$
\|g\|=\left\|g^{\prime}\right\|-\left\|g_{1 n}^{\prime 00}\right\|
$$

Для вычисления веса функции $g^{\prime}$ можно воспользоваться первой частью доказательства и формулой (7), аналогичные рассуждения проводятся и для функции $g_{1 n}^{\prime 00}$ 
Для случая, когда $f \in M_{3}$, докажем вторую часть теоремы. Для этого достаточно известную \#Р-полную задачу свести к нашей задаче. В качестве известной $\# P$-полной задачи рассмотрим задачу еnu $\left(\overline{x_{1}} \vee x_{2}\right)$ (см. [3]). Данную задачу можно сформулировать как задачу определения веса произвольной функции $g$ из $M_{3}$. Пусть $g\left(x_{1} \ldots, x_{n}\right) \in M_{3}$ и некоторые переменные в минимальной 2 -КНФ функции встречаются более трех раз. Построим функцию $f\left(x_{1}, \ldots, x_{n}\right)$ такую, что $\|f\|=\|g\|$ и все переменные в минимальной 2-КНФ функции $f$ встречаются не более трех раз. Пусть $x_{1}$ встречается $k$ раз в минимальной $2-\mathrm{KH} \Phi$ функции $g$ без отрицания и $l$ раз с отрицанием, $k+l \geqslant 4$, то есть

$$
g\left(x_{1}, \ldots, x_{n}\right)=\bigotimes_{i=1}^{k}\left(x_{1} \vee \overline{x_{p_{i}}}\right) \bigotimes_{j=1}^{l}\left(\overline{x_{1}} \vee x_{q_{j}}\right) h\left(x_{2}, \ldots, x_{n}\right)
$$

Для простоты будем полагать, что в минимальной 2-КНФ функции $g$ все остальные переменные встречаются не более трех раз. Положим $m=n+k+l$ и

$$
\begin{array}{r}
f\left(x_{1}, \ldots, x_{n}\right)=\bigotimes_{i=1}^{k}\left(\bar{x}_{p_{i}} \vee x_{n+i}\right){\underset{j=1}{\ell}\left(\bar{x}_{n+k+j} \vee x_{q_{j}}\right)}_{\underbrace{k+l-1}_{r=1}}^{\bigotimes}\left(\bar{x}_{n+r} \vee x_{n+r+1}\right) \\
\&\left(\bar{x}_{n+r} \vee x_{1}\right) \&\left(\bar{x}_{1} \vee x_{n+1}\right) \& h\left(x_{2}, \ldots, x_{n}\right) .
\end{array}
$$

Легко показать, что построенная в (8) КНФ является минимальной и в то же время каждая переменная в ней встречается не более трех раз.

Если для минимальной 2-КНФ функции $g$ существуют хотя бы две переменные, встречающиеся более трех раз, то мы можем продолжить описанную процедуру.

Рассмотрим общий случай. Пусть в 2-КНФ (5) все переменные встречаются не более трех раз. Выше мы выделили целый класс \#Р-полных индивидуальных подзадач задачи еnu $(2,3)$, поэтому и сама задача enu $(2,3)$ является \# $P$-полной. \#Pполнота задачи еnu $(2, r)$ при $r>3$ доказывается аналогично тому, как она доказывается для задачи еnu $(2,3)$. Теорема доказана.

Следующая теорема выделяет класс полиномиальных подзадач задачи еnu( 2,3$)$.

Теорема 7. Пусть построенная по системе (6) функция $f\left(x_{1}, \ldots, x_{n}\right) \in M_{3} u$ граф ее минимальной 2-KНФ $G(f)$ при ограничении на вершины $x_{1}, \ldots, x_{n}$ является выходящим деревом (входящим деревом, лесом из входящих и выходящих деревъев). Тогда данная подзадача задачи епu $(2, n)$ является полиномиалъной, и трудоемкость соответствующего алгоритма есть $O\left(n^{2}\right)$.

Доказателъство. Для доказательства теоремы достаточно построить соответствующий полиномиальный алгоритм. Пусть $G(f)-$ дерево, $x_{1}-$ его корень, $\operatorname{deg}\left(x_{1}\right)=k$, $x_{2}, \ldots, x_{k+1}$ смежны с $x_{1}, T_{1}, \ldots, T_{k}$ - подграфы $G(f)$, являющиеся корневыми деревьями с корнями соответственно в вершинах $x_{2}, \ldots, x_{k+1}$ (то есть максимальные по включению поддеревья, не содержащие $x_{1}$ ). Легко видеть, что выполняющим векторам функции $f$ соответствуют независимые в графе $G(f)^{T}$ подмножества вершин. Через $N(T)$ обозначим число независимых (не обязательно максимальных) подмножеств вершин транзитивного замыкания $T^{T}$ дерева $T$. Тогда

$$
N(G(f))=1+\prod_{i=1}^{k} N\left(T_{i}\right)
$$

Опишем следующую рекурсивную процедуру. 


\section{CYET $(T)$}

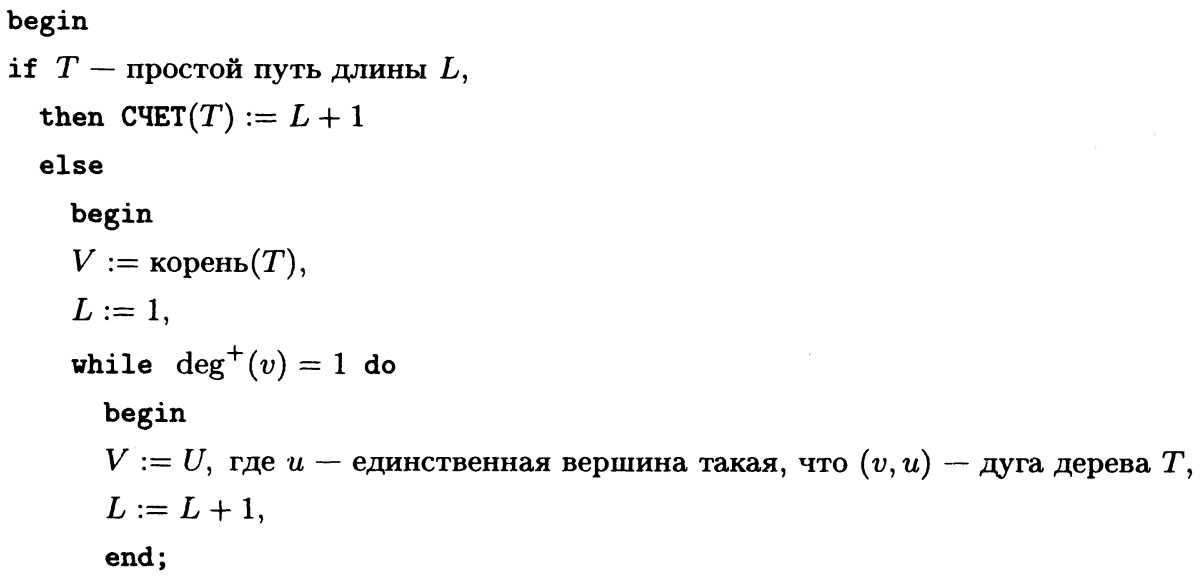

пусть $T_{1}, \ldots, T_{k}$ - выходящие из прямых потомков вершины $v$ поддеревья, тогда

$\operatorname{CYET}(T):=L+\prod_{i=1}^{k} \operatorname{CYET}\left(T_{i}\right)$

end;

end.

Заметим, что суммарное число рекурсивных вызовов в процедуре СчЕТ $(G(f))$ не превосходит числа $n$ вершин дерева, а при вычислении произведения также производится не более $n$ операций. Корректность алгоритма очевидна. Трудоемкость составит $O\left(n^{2}\right)$ элементарных операций. Теорема доказана.

Перейдем теперь к доказательству неравновероятности биюнктивных функций, существенно зависящих не менее, чем от пяти переменных.

Теорема 8. Пусть $f\left(x_{1}, \ldots, x_{n}\right)$ - произвольная биюнктивная функция, существенно зависящал от всех переменнъх, $n \geqslant 5$. Тогда функция $f\left(x_{1}, \ldots, x_{n}\right)$ неравновероятна и выполнено в точности одно из следующих двух условий:

- функция $f\left(x_{1}, \ldots, x_{n}\right)$ имеет вес, равный $2^{n-1}+1$ и эквивалентна бункции $\&_{i=2}^{n}\left(x_{1} \vee x_{i}\right)$ o epynne $Q_{n}$,

- вес фунхции $f\left(x_{1}, \ldots, x_{n}\right)$ строго менъше $2^{n-1}$.

Доказателъство. Достаточно доказать теорему для случая $f \in W P$. В противном случае достаточно выбрать произвольный вектор из $N_{f}$ и навесить отрицания на соответствующие переменные функции $f$. Без ограничения общности будем считать, что в минимальной КНФ функции $f$ переменная $x_{1}$ встречается максимальное число раз. Далее, считаем, что функция $f$ не имеет имплицент вида $x_{i}^{a}$, поскольку в этом случае очевидно, что теорема верна.

Представим функцию $f$ в виде

$$
f\left(x_{1}, \ldots, x_{n}\right)=\ell_{i=1}^{k}\left(\bar{x}_{1} \vee x_{p_{i}}\right) \bigotimes_{j=1}^{l}\left(x_{1} \vee x_{q_{j}}^{b_{j}}\right) h\left(x_{2}, \ldots, x_{n}\right) .
$$


Если $h\left(x_{2}, \ldots, x_{n}\right) \equiv 1$, то в формуле (9) получим, что $k+l \geqslant n-l$. Допустим, что $p_{i}=q_{j}$ при некоторых $i$ и $j$. Поскольку КНФ (9) минимальна, из равенства $p_{i}=q_{j}$ следует, что $b_{j}=0$. В этом случае $x_{1} \oplus x_{q_{j}} \oplus 1$ - имплицента функции $f$, но поскольку функция $f$ существенно зависит от всех переменных, в КНФ (9) есть сомножители, содержащие другие переменные. Значит, вес функции $f$ строго меньше веса функции $x_{1} \oplus x_{q_{j}} \oplus 1$, который равен $2^{n-1}$.

Будем теперь считать, что множества $M=\left\{p_{1}, \ldots, p_{k}\right\}$ и $N=\left\{q_{1}, \ldots, q_{l}\right\}$ не пересекаются. Тогда $k+l=n-l$. В этом случае легко видеть, что

$$
\|f\|=2^{k}+2^{n-k-1} \text {. }
$$

Максимум этого выражения, очевидно, равен $2^{n-1}+1$, причем мы попадаем в условия первого пункта теоремы, а остальные значения не превышают $2^{n-1}$.

Предположим теперь, что функция $h\left(x_{2}, \ldots, x_{n}\right)$ не равна тождественно единице. Аналогично доказанному выше будем считать, что множества $M=\left\{p_{1}, \ldots, p_{k}\right\}$ и $N=\left\{q_{1}, \ldots, q_{l}\right\}$ не пересекаются, так как в противном случае вес функции $f$ строго меньше $2^{n-1}$. Сначала рассмотрим случай $k=n-1$. Покажем, что в этом случае функция $f$ неравновероятна, а значит, ее вес строго меньше $2^{n-1}$. В самом деле, если $x_{i}^{a} \vee x_{j}^{b}-$ простая имплицента функции $h$, то легко видеть, что

$$
\|f\| \leqslant\left\|\bigotimes_{i=2}^{n}\left(\bar{x}_{1} \vee x_{i}\right) \&\left(x_{i}^{a} \vee x_{j}^{b}\right)\right\|<2^{n-1} .
$$

Аналогично рассматривается случай $l=n-1$. Теперь рассмотрим случай $k+l=n-1$, $k>0, l>0$. Тогда мы попадаем в условия уже доказанного утверждения.

Будем считать, что $k+l<n-1$. Тогда существует переменная $x_{m}$, которая не входит в произведение

$$
\bigotimes_{i=1}^{k}\left(\bar{x}_{1} \vee x_{p_{i}}\right) \ell_{j=1}^{l}\left(x_{1} \vee x_{q_{j}}^{b_{j}}\right) \text {. }
$$

Это означает, что ни одна из функций $\bar{x}-1 \vee x_{m}, x_{1} \vee x_{m}, x_{1} \vee \bar{x}_{m}, \bar{x}_{1} \vee \bar{x}_{m}$ не является имплицентой функции $f$.

Сначала рассмотрим случай $k+l \geqslant 3, k \geqslant 1, l \geqslant 1$. В этом случае будем считать, что $k \geqslant 2$. Тогда

$$
\|f\| \leqslant\left\|\left(x_{1} \vee x_{2}\right)\left(x_{1} \vee x_{3}\right)\left(\bar{x}_{1} \vee x_{4}\right)\right\|=6 \cdot 2^{n-4}<2^{n-1},
$$

и теорема верна.

Пусть теперь $l=0, k \geqslant 2$. Тогда существует переменная $x_{m}$, не входящая в произведение $\&_{i=1}^{k}\left(\bar{x}_{1} \vee x_{p_{i}}\right)$. В этом случае имплицентами функции $f$, с точностью до навешивания отрицаний на переменные, не входящие в указанное произведение, могут быть дизъюнкты $x_{m} \vee x_{p_{j}}, x_{m} \vee \bar{x}_{p_{j}}, x_{m} \vee x_{q}$. В первом случае

$$
\|f\| \leqslant\left\|\left(x_{1} \vee x_{2}\right)\left(x_{1} \vee x_{3}\right)\left(x_{2} \vee x_{4}\right)\right\|=2^{n-1} \text {. }
$$

Однако, поскольку функция $f$ задана минимальной КНФ и зависит существенно по крайней мере от пяти переменных, в ее КНФ входит по крайней мере еще один дизъюнкт кроме трех указанных выше, и неравенство будет строгим. Рассмотрим второй случай. Тогда

$$
\|f\| \leqslant\left\|\left(x_{1} \vee x_{2}\right)\left(x_{1} \vee x_{3}\right)\left(\bar{x}_{2} \vee x_{4}\right)\right\|=7 \cdot 2^{n-4}<2^{n-1}
$$


и теорема верна. В третьем же случае

$$
\|f\|=\left\|\left(x_{1} \vee x_{2}\right)\left(x_{1} \vee x_{3}\right)\left(x_{4} \vee x_{5}\right)\right\|=15 \cdot 2^{n-5}<2^{n-1}
$$

Осталось рассмотреть случаи $k=l, l=0$ и $k=l=1$. Поскольку $x_{1}$ - переменная с наибольшим числом вхождений в КНФ из правой части (9), в первом случае каждая переменная входит в 2-КНФ в точности по одному разу. Это значит, что $n$ четно, и с помощью преобразования из $Q_{n}$ из функции $f$ можно получить функцию $\left(x_{1} \vee x_{2}\right)\left(x_{3} \vee x_{4}\right) \ldots\left(x_{n-1} \vee x_{n}\right)$. Значит $\|f\|=3^{n / 2}<2^{n-1}$ при четных $n \geqslant 5$. Если же $k=l=1$, то каждая переменная встречается в КНФ из (9) не более двух раз. Для этого случая можно воспользоваться результатами доказательства теоремы 6 и формулой (7). Теорема доказана.

Функцию $f\left(x_{1}, \ldots, x_{n}\right)$ будем называть дважды биюнктивной, если она биюнктивна и биюнктивна функция $f \oplus 1$ (см. [3]). На основании доказанной теоремы приведем более короткое, чем в [3], доказательство следующего утверждения.

Следствие 2. Не существует дважды биюнктивных функций, существенно зависящих более, чем от четырех переменных.

Доказателъство. Пусть $f\left(x_{1}, \ldots, x_{n}\right)$ - дважды биюнктивная функция. Тогда функция $f \oplus 1$ биюнктивна. Если $n>4$, то $\|f\| \neq 2^{n-1}$. Предположим, что $\|f\|<2^{n-1}$. Так как функция $f \oplus 1$ биюнктивна и $\|f \oplus 1\|+\|f\|=2^{n}$, по теореме $8\|f \oplus 1\|=$ $2^{n-1}+1$. Это означает, что, с точностью до перестановки переменных и навешивания отрицаний,

$$
f \oplus 1=\bigotimes_{i=2}^{n}\left(x_{1} \vee x_{i}\right)
$$

Тогда можно указать три выполняющих вектора функции $f$, не удовлетворяющих критерию биюнктивности из [3]:

$$
a_{1}=(0,1,1,0,1, \ldots, 1), \quad a_{2}=(0,1,0,1,1, \ldots, 1), \quad a_{3}=(0,0,1,1,1, \ldots, 1) .
$$

Случай $\|f \oplus 1\|<2^{n-1}$ рассматривается аналогично. Следствие доказано.

Заметим, что существуют равновероятные дважды биюнктивные функции от трех и четырех переменных (см. [6]).

В заключение отметим, что в статье изложен эффективный метод минимизации булевых биюнктивных функций, получены верхние оценки для длины минимальных 2-КНФ. Кроме того, оценена сложность задачи расчета веса биюнктивной функции, заданной 2-КНФ, доказана неравновероятность биюнктивных функций, существенно зависящих от пяти и более переменных. 


\section{Список литературы}

1. Schaefer T., Complexity of satisfiability problems. Proc. 10th Annual ACM Symp. on Theory of Computing, 1978, p. 216-226.

2. Данцин Е. Я., Алгоритмика задачи выполнимости. Вопросы киберн. (1987) 131, 7-21.

3. Горшков С. П., Применение теории $N P$-полных задач для оценки сложности решения булевых уравнений. Обозрение прикладной и промычленной математики (1995) 2, №3, 325-398.

4. Горшков С. П., О сложности задачи нахождения числа решений систем булевых уравнений. Дискретная математика (1996) 8, №1, 72-85.

5. Горшков С. П., О пересечении классов мультиаффинных, биюнктивных, слабо положительных и слабо отрицательных булевых функций. Обозрение прикладной и промышленной математики (1997) 4, №2, 238-259.

6. Гизунов С. А., Носов В. А., О классификации всех булевых функций 4-х переменных по классам Шефера. Обозрение прикладной и промышленной математики (1995) 2, №3, 440-467.

7. Харари Ф., Теория графов. Мир, Москва, 1973.

8. Ахо А., Хопкрофт Дж., Ульман Дж., Построение и анализ вычислительных алгоритмов. Мир, Москва, 1979.

9. Кристофидес Н., Теория графов. Алгоритмический подход. Мир, Москва, 1979.

10. Гэри М., Джонсон Д., Вычислителъные машины и труднорешаемые задачи. Мир, Москва, 1982.

11. Яблонский С. В., Введение в дискретную математику. Наука, Москва, 1979.

Статья поступила 10.10.2000. 